\title{
Philosophiques
}

\section{Le processus contre-transférentiel comme travail de deuil}

\section{Lise Monette}

Volume 4, numéro 2, octobre 1977

Philosophie et psychologie

URI : https://id.erudit.org/iderudit/203081ar

DOI : https://doi.org/10.7202/203081ar

Aller au sommaire du numéro

Éditeur(s)

Société de philosophie du Québec

ISSN

0316-2923 (imprimé)

1492-1391 (numérique)

Découvrir la revue

Citer cet article

Monette, L. (1977). Le processus contre-transférentiel comme travail de deuil. Philosophiques, 4(2), 305-312. https://doi.org/10.7202/203081ar d'utilisation que vous pouvez consulter en ligne.

https://apropos.erudit.org/fr/usagers/politique-dutilisation/ 


\title{
LE PROCESSUS CONTRE-TRANSFERENTIEL COMME TRAVAIL DE DEUIL*
}

\author{
par Lise Monette
}

J'en suis à mon troisième colloque ...

Le premier, imaginaire, portait sur les rapports de la psychanalyse et du marxisme à l'aide de Castel, Psychanalyse et politique et Marxisme-léninisme et psychanalyse. Une autocritique provoqua un premier deuil. Je ne pouvais malheureusement que seriner les thèses de ses auteurs. Un bénéfice de perdu: je ne pourrais brandir l'étendard de la gauche à propos de la psychanalyse!

Le second, imaginaire, portait sur les ratés du discours philosophique et la place de la question dans sa tradition : place thétorique, pédagogique, stratégique ... pensais-je ; et enfin l'occultation des lieux de la jouissance du philosophe. Autocritique, cette fois, devant la complexité du sujet auquel je ne faisais guère justice et qui nécessite de reprendre presque l'ensemble de l'histoire de la philosophie. Deuil, cette fois, de la jonction de ma pratique philosophique et psychanalytique.

Et maintenant devant vous je dis:

"Celui qui dit, par son dit, s'interdit la jouissance, ou corrélativement, celui qui jouit fait toute lettre - et tout dit possible - s'évanouir dans l'absolu de l'annulation qu'il célèbre. "

\section{S. LECLAIRE}

De quels lieux la psychanalyse interroge-t-elle, interpelle-telle la psychologie ? J'ai élu de parler à partir de deux lieux prévilégiés de la psychanalyse : la jouissance et la castration. Précisons ces concepts autour desquels se situe cette intervention qui subvertit consciemment l'enjeu de cette table ronde sur " la contestation DE la psychanalyse ", en substituant au DE génitif le DE de l'ablatif

\footnotetext{
* Ce texte demeure conforme à sa forme initiale tributaire d'une intervention didactique dans sa visée et limitée dans le temps.
} 
pour m'adresser à un interlocuteur privilégié : le psychologue. Ce court essai de circonstance se marque, oscille donc entre deux pôles coextensifs l'un de l'autre : la castration et la jouissance.

La castration est un registre auquel on ne peut échapper. $P$. Castoriadis la définit ainsi : "La découverte dans le registre identificatoire qu'on n'a jamais occupé la place qu'on a cru sienne et que, à l'opposé, on est censé occuper une place à laquelle on ne peut pas encore être. "La castration est ici envisagée comme décentrement inévitable du sujet, de la parole, du discours. Elle réfère également aux failles et ratés de tout discours. "Le propre du champ psychanalytique (selon Lacan) est de supposer que le discours du sujet se développe normalement dans l'ordre de l'erreur, de la méconnaissance, voire de la dénégation - ce n'est pas tout à fait le mensonge, c'est entre l'erreur et le mensonge. ${ }^{2}$ Il souligne que la vérité ne peut se déployer que là où le mensonge est possible.

La jouissance, à la différence du plaisir, est imprévisible. Barthes, au sujet de la lecture, distingue des textes de plaisir qui contentent et captivent, et ne rompent en rien avec la culture, et des textes de jouissance qui " mettent en état de perte et font vaciller les assises historiques, culturelles... et mettent en crise le rapport au langage $"{ }^{3}$ La jouissance y est décrite comme évanouissement, interdit, à savoir qu'elle ne peut être dite qu'entre les lignes.

Je désire interpeller la psychologie à partir de ce que la psychanalyse nous dévoile du processus contre-transférentiel dans le déroulement de la cure et que la première néglige. Freud décrit l'attention flottante de l'analyste comme pendant aux associations libres de l'analysant, le fameux " listening with the third ear " de Reik, - ces expressions au demeurant fort obscures sur le travail en cause. Je tenterai de cerner comment peut s'accomplir cette écoute à travers l'analyse des deuils que l'analyste effectue pour atteindre cette écoute. Finalement, je me pencherai brièvement sur la question des lieux psychiques de jouissance de l'analyste.

Dans " Deuil et mélancolie ", Freud décrit le deuil comme un retrait de libido des "liens qui la retiennent à l'objet ", en général

1. CASTORIADIS-AUlagnier, P., La violence de l'interprétation, P.U.F., 1975, p. 198.

2. LACAN, J., " Le Séminaire ", livre I, Les Écrits Techniques de Freud, Seuil, 1975, p. 291.

3. BARTHES, R., Le plaisir du texte, Seuil, 1973, p. 25. 
suite à la perte de l'objet. Les analystes ont donc écrit sur le deuil à effectuer en fin de cure. - Je n'en parlerai point. Ce qui sera en jeu ici, c'est le travail de deuil comme pratique de libération sur le plan économique d'énergie psychique normalement tournée vers l'objet d'abord, le moi ensuite, et mise par ce processus au service de l'inconscient afin de favoriser une écoute qui n'a rien à voir avec la compréhension empathique de Rogers ou l'identification massive au patient.

Ce deuil n'implique en aucune façon la tristesse. Il réfère à une conscience perdue pour elle-même parce qu'entièrement préoccupée de l'autre. Captive, mais non aliénée. Elle n'a plus son lieu propre en elle-même.

On peut sans doute supposer, à la suite d'Abraham, qu'un des effets du processus de deuil sera justement l'introjection de l'objet substitut de l'investissement d'amour initial.

Six champs d'exploration possibles du travail de deuil de l'analyste seront suggérés :

1- Serge Leclaire, dans une conférence inédite l'année dernière au Allan Memorial, soulignait la nécessité pour l'analyste d'écouter l'analysant en mettant entre parenthèse son SAVOIR THÉORIQUE, sorte d'epochè par laquelle l'écoute, au lieu de partir du confort de l'universel pour y ramener le particulier, s'en tient au HIC ET NUNC de la parole dans sa spécificité et son originalité propre.

Le statut de l'analyste, de sujet supposé savoir, indique le pivot de la relation transférentielle pour l'analysant, c'est-à-dire le mirage nécessaire à l'élaboration de fantasmes projectifs. Ce mirage, l'analyste aussi doit s'en extraire dans la mesure où le rabattement sur le corpus psychanalytique se révèle défensif, c'est-àdire dans la proportion où il l'utilise pour précéder et dominer le discours du patient et ainsi éviter l'angoisse de l'ignorance. C'est en ce sens précis que j'entends cette " passion de l'ignorance " dont parle Lacan et que je l'applique ici au travail de deuil nécessaire à une écoute analytique. En complément à celui-ci, peut-être faudrait-il radicaliser ce processus jusqu'à y inclure l'oubli à chaque séance du matériel précédent, c'est-à-dire la renonciation à la prévisibilité des énoncés en fonction des énoncés antérieurs, compulsion de répétition à laquelle la clinique démontre que le 
patient ne peut échapper. Mais l'analyste peut, à travers ce processus de deuil, rechercher la surprise, la petite différence, les " phénomènes résiduels " ${ }^{4}$ comme dit Freud, qui modulent toute répétition en évitant le double confort du savoir théorique et du savoir clinique, travail de deuil sur l'idéal du moi de l'analyste, c'est-à-dire sur la représentation que le moi se fait de son statut idéal de clinicien, c'est-à-dire de théoricien. Idéal du moi non sans rapport, d'ailleurs, avec celui que véhicule l'institution psychanaly. tique à laquelle il appartient.

2- "Quelque ambition thérapeutique que l'on nourrisse, l'expérience ne nous offre là que déceptions. "s L'accent, dans cette citation de Freud, porte sur les déceptions à éviter en faisant le DEUIL DE SES VISEEES THÉRAPEUTIQUES. Par delà l'économie d'un affect désagréable, s'énonce une conception de la cure comme pure recherche de vérité. Les implications pratiques de cette approche sont capitales.

Où gît le problème lorsque l'analyste souhaite la guérison de son patient? Par delà les bonnes intentions de l'analyste, nous découvrons SA conception de l'équilibre, SA conception de la normalité. La thérapie américaine axée sur l'adaptation du patient à son milieu en découle naturellement ; émerge alors aussi l'ego psycho$\log y$ où le thérapeute se présente comme moi auxiliaire du patient, béquille, prothèse ; et à l'intérieur de la psychanalyse même apparaissent des concepts comme "l'expérience corrective" d'Alexander. Est-il alors besoin d'insister davantage sur l'enjeu dans ce deuil des visées thérapeutiques?

3- Toute relation transférentielle et contre-transférentielle constitue une relation d'amour. Oserais-je parler de DEUIL DE L'INVESTISSEMENT D'OBJET, en l'occurrence de l'attachement affectif à l'analysant? Le travail avec des adolescents met particulièrement en évidence la nécesșité de ce deuil. Les interruptions de leur part qui se présentent comme rupture ne peuvent être interprétées ou même tout simplement donner naissance à une reprise, une relance que dans la mesure où l'analyste effectue, élabore à chaque fois le deuil de son affection pour l'adolescent ;

4. FreUd, S., "Analyse terminée et analyse interminable ", in Revue Française de Psychanalyse, XI, 1939 , p. 15.

5. FreUd, S., Idem, p. 17. 
celle-ci ne pouvant être perçue que comme un poids aussi lourd à porter que l'amour de ses parents dont justement il tente de se distancer.

Plus un analyste tient à conserver un patient, plus les chances sont grandes qu'il le quitte. L'apprenti analyste l'apprend à ses dépens. La diffraction de l'affection de l'analyste entre plusieurs patients favorise d'ailleurs la distance nécessaire à son élaboration et à celle de l'analysant. Le travail de deuil sur l'affection, contrairement à ce que l'on pourrait croire, ne crée pas l'élimination de toute implication émotionnelle (l'amour ne se tue pas à volonté), mais la rigueur, la rectitude du coeur dont l'envers serait une complaisance malsaine dans un jeu de séduction réciproque ou de délire à deux.

4- Le DEUIL DE SA CURIOSITÉ : soit de façon ponctuelle, lorsque l'analysant n'arrive pas à raconter une action, un fantasme présent ou passé, soit de façon plus globale dans le désir de tout savoir de la vie et surtout des pensées du patient. Curiosité dévorante qui abrite en son sein un désir mégalomaniaque de possession de l'autre.

C'est Marguerite Mahler, je crois, qui souligne l'importance du secret dans le processus d'individuation de l'enfant, dans la construction de son moi. Il y a des facettes d'un analysant que l'analyste ne connaîtra jamais. Mais à travers les résistances du patient à confier à un autre ses secrets, Fedida nous rappelle que la "perte d'un secret équivaut à l'évanouissement d'une parole".

5. Rarement dans la vie quotidienne voit-on le tabou de l'inceste mis en question, tellement il fait partie des lois intériorisées par la majorité dans notre culture. Ainsi en est-il chez les analystes du TABOU DES RELATIONS SEXUELLES avec les patients. Cela n'empêche pas la nécessité du travail de deuil, en particulier avec les patients hystériques. Le livre d'Erika Kaufmann, Le Transfert, l'illustre spectaculairement. Il faudrait reprendre ici en détail la discussion des premiers analystes autour de la frustration nécessaire à la dynamique de la cure. ${ }^{6}$

Le rabattement sur le réel que constitue la relation sexuelle se révélerait défensif par rapport à la création d'un espace imagi-

6. Celie-ci entendue dans un sens plus large et techniquement plus précis que dans ce texte-ci. 
naire favorable à l'élaboration de fantasmes. Un tel passage à l'acte constitue un rejet des conditions d'apparition du transfert : à savoir la résistance à la déréalisation que provoquent la position couchée du patient et la non-visibilité de l'analyste.

6- LE DEUIL DE LA PAROLE, pour l'analyste, implique qu'il reconnaisse que le véritable maître est l'analysant ; que ses paroles importunes ne peuvent que distraire le patient de ses propres associations. Ce qui soulève le problème des interventions nécessaires; nécessaires à la levée de résistances, nécessaires à la construction ou reconstruction d'un ensemble de fantasmessouvenirs : point d'ancrage de l'histoire du patient ; ce qui soulève surtout peut-être la question d'interventions gratuites (jeu de mots, métaphores, association d'éléments en apparence disparates), gratuites en ce sens qu'elles constituent une pure relance du discours de l'autre mais surtout une effraction de l'AUTRE.

À la limite, cette parole de l'analyste tant désirée et tant crainte par l'analysant n'implique-t-elle pas un viol, une violence incontournable de l'inconscient dans ce qu'il débloque d'affects envahissants? Deuil d'une parole si puissante en ses effets que le silence dominant transforme la moindre intervention en discours performatif.

\section{Comme l'écrit Viderman :}

"L'analyste ne peut dire ce qui ferait le plus de sens parce que le sens n'est pas seul à trancher de ce qu'il sera ou non entendu. Il ne suffit pas que l'interprétation porte le sens, il faut que quelqu'un d'autre le reçoive au même instant avec le moins d'altération possible. A chaque moment l'analyste doit tenir compte de l'état affectif du patient ; du degré du transfert ; du degré de résistance ; de l'a. vancement de la cure, des prises de conscience déjà effectuées, de leurs qualités; du travail et du temps nécessaires pour que le patient les fasse siennes. " 7

Furent pointés tour à tour dans ce texte sur un des processus contre-transférentiels : le deuil du savoir, de la visée thérapeutique, de l'attachement, de la curiosité, de la relation sexuelle et de la parole. Pour paraphraser la citation initiale de P. Castoriadis : autant de découvertes par l'analyste qu'il n'occupe pas la place que le patient a cru la sienne, qu'IL a cru lui aussi être

7. Viderman, S., La Construction de l'espace analytique, Denoël, 1970, p. 48. 
sienne ! Le choix de ce sujet tient au destinataire imaginaire de ce discours : la psychologie qui aborde dans la méconnaissance cette question du rapport du thérapeute à son patient. Dans le meilleur des cas, est évoquée à la manière rogerienne une problématique relation fusionnelle dont le leurre n'a d'égal que celui des amoureux qui rêvent d'être plus qu'un pour se découvrir deux, comme le souligne Lacan.

Mais une fois terminée cette énumération qui ne se veut d'ailleurs pas exhaustive, une question demeure : qu'en est-il de la jouissance de l'analyste?

Parler d'amour est en soi jouissance, nous dit Barthes dans le Plaisir du texte. Il y a un PLAISIR VOYEURISTE non équivoque dans le voilement-dévoilement que constitue le discours de l'analysant. Le premier des plaisirs, selon Aristote dans l'Éthique à Nicomaque est le voir, l'entendre n'apparaissant qu'en troisième lieu. Freud, à l'origine, se plaça derrière ses patients parce qu'il avait horreur de se faire dévisager, lui dont tous les patients et collaborateurs témoignent du regard perçant. Les thérapeutes qui huit heures par jour font face à leurs patients partagent l'avis de Freud. Cela n'exclut pas l'hypothèse réjouissante que l'analyste se terre pour mieux jouir...

Le deuil de l'investissement d'amour permet de concevoir sur le plan économique un double destin à l'énergie ainsi libérée, l'introjection, d'une part, et, d'autre part, la SATISFACTION SADIQUE, issue des ambivalences de l'analyste vis-à-vis de son patient. Le sadisme de certains silences, de certaines paroles, mais surtout, faut-il le dire, la satisfaction à l'occasion de ne pas se trouver empêtré dans les problèmes et souffrances de l'autre.

- Last but not least - à propos dè ce triptyque sur la jouissance: la RECONNAISSANCE dans la double acception du terme adressée du patient à l'analyste pour "service rendu ", comme l'indique le langage populaire et dans son versant hégélien par sa création de l'analyste dans la position de maitre, de figure parentale idéalisée. Jouissance également non négligeable de la reconnaissance scientifique que lui procurent ses pairs.

Rétrospectivement, il est frappant de constater que les jouissances de l'analyste agissent à la manière de retours du refoulé 
suite aux deuils successifs qui paradoxalement frayent les voies à ces jouissances.

En guise de conclusion, deux évocations de l'aventure analytique que me suggèrent ces réflexions :

La première : le titre d'un film de la nouvelle vague anglaise des années '60: "The Loneliness Of The Long Distance Runner ", la solitude du coureur de fond...

La seconde : celle de Thérèse d'Avila et de Jean de la Croix. Eh oui! les mystiques sont ceux qui ont su le mieux écrire la jouissance et l'extase, comme le soulignent Lacan et Kristeva.

Et pour boucler la boucle de cette esquisse sur le processus contre-transférentiel de deuil :

"Celui qui dit, par son dit, s'interdit la jouissance "...

\section{S. LECLAIRE}

Département de Philosophie, Université du Québec à Montréal. 